\title{
Estudio de Línea Base de las Concentraciones de Metales Pesados en Chocolate en Polvo
}

\section{Baseline Study of Heavy Metal Concentrations in Powdered Chocolate Marketed in Guayaquil, Ecuador}

\begin{abstract}
Cristhian Enrique Sánchez-Soledispa ${ }^{I}$, Diana Carolina Zambrano-Vera ${ }^{1}$, Olga Raquel Arévalo-Castro ${ }^{\text {}}$, Vinicio Xavier

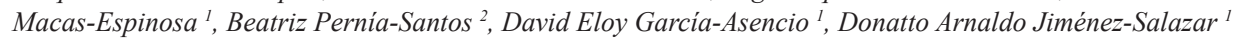

INFORMACIÓN DEL

\section{ARTÍCULO}

Fecha de recepción: diciembre 11,2020 Fecha de aceptación: enero 29, 2021.

1 Facultad de Ciencias Naturales, Universidad de Guayaquil, Ecuador. E-mail: cristhianss95@gmail.com Código ORCID:

https://orcid.org/0000-0002-1698-226 E-mail: dianacaro_zamve@hotmail.com Código ORCID:

https://orcid.org/0000-0003-2800-2158

E-mail: olga.arevaloc@ug.edu.ec

Código ORCID:

https://orcid.org/0000-0001-9040-2721

E-mail: Vinicio.macase@ug.edu.ec Código ORCID:

https://orcid.org/0000-0003-0319-0631

E-mail: david.garciaa@ug.edu.ec

Código ORCID:

https://orcid.org/0000-0003-3836-9519

E-mail: scofingarnold@gmail.com

\section{Resumen}

En Ecuador se ha demostrado contaminación por metales pesados en el cacao; sin embargo, no se ha estudiado el impacto de esta contaminación sobre el chocolate en polvo de consumo nacional. El objetivo de este estudio fue generar una línea base de la concentración de arsénico, cadmio y plomo en chocolate en polvo comercializado en la ciudad de Guayaquil. Para ello, se adquirieron muestras de chocolate en las zonas norte, centro y sur de la ciudad en tiendas y supermercados. Se eligieron tres marcas más populares y se analizaron por espectrofotometría de absorción atómica con horno de grafito. El plomo no fue detectable en el chocolate; el As se encontró en bajos niveles en la Marca-1 $(0,030 \pm 0,021 \mathrm{mg} / \mathrm{kg})$ y la Marca-3 $(0,060 \pm 0,034 \mathrm{mg} / \mathrm{kg})$, y, por el contrario, se halló cadmio en todas las marcas de chocolate, con concentraciones de $0,236 \pm 0,082 \mathrm{mg} / \mathrm{kg}$ en la Marca-1, 0,169 $\pm 0,066 \mathrm{mg} / \mathrm{kg}$ en la Marca-2 y la Marca-3 superó el límite máximo permisible de $0.600 \mathrm{mg} / \mathrm{kg}$ según normativa europea con $1,440 \pm 0,212 \mathrm{mg} / \mathrm{kg}$, representando un riesgo para los consumidores.
\end{abstract}

Código ORCID:

https://orcid.org/0000-0002-4197-5598

2 Instituto de Investigaciones de Recursos Naturales. Universidad de Guayaquil, Ecuador.

E-mail: beatriz.pernias@ug.edu.ec Código ORCID:

https://orcid.org/0000-0002-2476-7279

CITACIÓN: Sánchez-Soledispa, C.E., Zambrano-Vera, D.C., Arévalo-Castro, O.R., Macas-Espinoza, V.X., PerníaSantos, B., García-Asencio, D.E. \& Jiménez-Salazar, D.A. (2021). Estudio de Línea Base de las Concentraciones de Metales Pesados en Chocolate en Polvo Comercializado en Guayaquil, Ecuador. Investigatio, 16, 62-77. doi:10.31095/investigatio.2021.16.6

\section{ENLACE DOI:}

http://dx.doi.org/10.31095/investigatio. 2021.16 .6

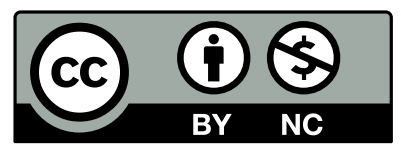

\section{Palabras Clave:}

Cacao, contaminación, metales pesados.

Clasificación JEL: I110.

\begin{abstract}
Heavy metals contamination has been demonstrated in cocoa in Ecuador. The impact of this contamination on powdered chocolate for national consumption has not, however, been studied. This study's objective was to generate a baseline of arsenic, cadmium and lead concentration in powdered chocolate marketed in Guayaquil. To execute this study, chocolate samples were acquired in the north, center and south of the city in stores and supermarkets. The three most popular brands were chosen and analyzed by atomic absorption spectrophotometry with a graphite furnace. Lead was not detectable in the chocolate; arsenic was found at low levels in Brand-1 (0.030 $\pm 0.021 \mathrm{mg} / \mathrm{kg})$ and in Brand $-3(0.060 \pm 0.034 \mathrm{mg} / \mathrm{kg})$. On the contrary, cadmium was found in all chocolate brands, with concentrations of $0.236 \pm$ $0.082 \mathrm{mg} / \mathrm{kg}$ in Brand-1 and $0.169 \pm 0.066 \mathrm{mg} / \mathrm{kg}$ in Brand -2 . Brand -3 exceeded the maximum permissible limit $0.600 \mathrm{mg} / \mathrm{kg}$ according to European regulations with $1.440 \pm 0.212 \mathrm{mg} / \mathrm{kg}$, representing a risk for consumers.
\end{abstract}

\section{Keywords:}

Cocoa, contamination, heavy metals.

JEL Classification: I110.

\section{2}

INVESTIGATIO No. 16, Marzo 2021, pp. 62-77

(C) Universidad Espíritu Santo - UEES

ISSN: 1390-6399 ISSN-e: 2602-8336 


\section{Introducción}

El cacao es uno de los productos más sobresalientes en el mercado a nivel mundial, por su fácil manejo, y esto a su vez ha originado la presencia de metales principalmente en los suelos, los mismos que se han descrito según su fuente: natural o antrópico; Sin embargo, se ha registrado metales en las plantas a través de sus raíces, ya que absorben estos metales pudiendo acumularlo en las diferentes estructuras y cantidades principalmente en sus semillas y hojas (Casteblanco, 2018). Dentro el continente Americano, el cultivo de cacao se extiende desde México a Brasil con un $40 \%$, Colombia $9 \%$ Ecuador con un $24 \%$, de hectáreas sembradas obteniendo juntos 235 mil toneladas al año y en África en países como Costa de Marfil, Ghana, Indonesia, Camerún son los principales países productores del mundo ya que juntos obtienen alrededor de 2.752 mil toneladas al año (FAO, 2018).

Dentro de los productores, Ecuador posee un cacao muy apreciado mundialmente por su sabor y aroma, siendo estas variables claves para la producción de chocolates de excelente calidad a nivel mundial (Wong, 2017). Según Anecacao (2015) la agricultura es la segunda actividad de exportación más importante dentro del país después de la industria petrolera, siendo Ecuador el cuarto mayor productor de cacao en el mundo, exportando el $87 \%$ de su producción como grano de cacao a Europa, principalmente Francia, Alemania e Inglaterra, así como a Estados Unidos.
Por otro lado, las semillas de Theobroma cacao (familia Malvaceae), se utilizan para hacer licor de chocolate, manteca de cacao, chocolates y chocolate en polvo (de Sousa et al., 2018; Maddela et al., 2020). Sin embargo, existe una reciente preocupación internacional con respecto a la presencia de trazas de metales en los tejidos de cacao y sus derivados. Estudios recientes han demostrado que metales pesados como el arsénico, bismuto, cromo, cadmio y plomo, se pueden acumular en los granos de cacao, cáscaras de las mazorcas, y en productos a base de cacao (Huamani et al., 2012; Chavez et al., 2015; Bertoldi et al., 2016). Las presencias de trazas de estos elementos provienen de fuentes naturales como el suelo y las erupciones volcánicas, y artificiales por el uso de fertilizantes y pesticidas en la industria agrícola (Mite et al., 2010; Gramlich et al. (2018); Maddela et al., 2020). Además, se puede mencionar que el árbol del cacao puede absorber los metales pesados del suelo y acumularlos en sus tejidos, representando una fuente de contaminación del chocolate, así como también puede ocurrir contaminación en el proceso de fabricación (Abt et al., 2018).

Se ha descrito en numerosos trabajos que la semilla de cacao en Ecuador puede contener metales pesados. Según Mite, Carrillo, \& Durango (2010), las concentraciones máximas encontradas en las almendras de cacao supera los 2,000 $\mathrm{mg} / \mathrm{kg}$ de Cd en las provincias de El Oro, Guayas, Manabí, Orellana, Napo y Zamora Chinchipe. Según estos autores, El Oro presenta los valores más altos de cadmio en cacao $(4,080 \mathrm{mg} / \mathrm{kg}$ de $\mathrm{Cd})$, 
seguido por Guayas y Manabí con 3,570 y $3,460 \mathrm{mg} / \mathrm{kg}$, respectivamente. En otros estudios más recientes se ha demostrado que en el sur de Ecuador, los granos de cacao mostraron un promedio de 0,940 $\mathrm{mg} / \mathrm{kg}$ de $\mathrm{Cd}$ y el $66 \%$ de las muestras excedieron el umbral de $0,600 \mathrm{mg} / \mathrm{kg}$ propuesto por la Unión Europea (Chavez et al., 2015). Así mismo, en otra investigación analizaron el cacao en seis provincias de Ecuador y presentaron un promedio de cadmio de $0,970 \mathrm{mg} / \mathrm{kg}$ (Barraza et al., 2017). En el mismo sentido, Argüello et al., (2019) analizaron 254 muestras de cacao ecuatoriano y hallaron niveles de $\mathrm{Cd}$ de 0,030 a 10,400 $\mathrm{mg} / \mathrm{kg}$ con una media geométrica de $0,550 \mathrm{mg} / \mathrm{kg}$ y una media aritmética de $0,900 \mathrm{mg} / \mathrm{kg}$ de $\mathrm{Cd}$, donde el $45 \%$ de las muestras superaron el límite permisible. Se ha sugerido que la contaminación del cacao se debe al $\mathrm{Cd}$ presente en el suelo de manera natural por la actividad volcánica, al uso de fertilizantes y al agua para riego contaminada con este metal pesado (Mite et al., 2010; Chávez et al., 2015; Chávez et al., 2016; Barraza et al., 2018; Kruszewski et al., 2018; Furcal-Beriguete \& Torres-Morales, 2020; Argüello et al., 2021).

Dentro de los metales pesados se han descrito el arsénico, el cadmio y el plomo como los más tóxicos presentes en el cacao (Abt et al., 2018; Anyimah-Ackah et al., 2018), y de los derivados del cacao el chocolate en polvo es uno de los productos más consumidos y en especial por los niños, donde la presencia de metales pesados representa un riesgo para su salud y una preocupación pública (Abt et al., 2018; Anyimah-Ackah et al., 2018;
Maddela et al., 2020). El arsénico es un metaloide que se ha asociado con melanosis, hiperqueratosis, cáncer de vejiga, mama, laringe y pulmón, diabetes tipo 2, anemia, daño renal y disminución en el rendimiento cognitivo (Mandal \& Suzuki, 2002; Maiti et al., 2012; Khan et al., 2020). El cadmio se ha descrito como un compuesto cancerígeno, mutagénico y teratogénico, que genera diabetes, anemia, daño renal, hipertensión y osteoporosis (García et al., 2012; Flora \& Agrawal, 2017; Hamid et al., 2019) y el plomo se ha asociado con daño a los sistemas reproductivos, nerviosos, cardiovascular y hematopoyético, es genotóxico y cancerígeno (Devóz et al., 2017; Chen et al., 2019; Ahn et al., 2020).

En este sentido, en algunos estudios realizados en países como Malasia (Mounicou et al., 2003), Perú (Laila et al., 2019), Venezuela (Mounicou et al., 2003), Latinoamérica y África (Abt et al., 2018), se han detectado altos niveles de cadmio y plomo en el chocolate en polvo, sin embargo, se desconocen los niveles de estos metales en el chocolate en polvo de venta en Ecuador.

A tenor de lo sustentado, se planteó la hipótesis de que el chocolate en polvo comercializado en Guayaquil podía contener elevados niveles de metales pesados, en especial de cadmio. Es por ello que, el objetivo de este estudio fue generar una línea base de la concentración de arsénico, cadmio y plomo en el chocolate en polvo expendido en la ciudad de Guayaquil, observar si existían diferencias en el contenido de metales entre diferentes 
marcas, precios, origen y zonas de la ciudad, así como comparar las concentraciones con los límites máximos permisibles para consumo humano y proponer algunas medidas para mitigar la contaminación en el chocolate en polvo.

\section{Materiales y métodos}

\section{Área de Estudio}

Ecuador se encuentra en Suramérica, en las costas del Océano Pacífico, en la zona ecuatorial. La segunda ciudad más importante del país es Guayaquil y es la más importante a nivel comercial (Alcaldía de Guayaquil, 2016). Guayaquil está ubicada en la provincia de Guayas, en la región costa del Ecuador continental (Figura 1). Esta ciudad está ubicada en la margen occidental del río Guayas $\left(79^{\circ} 50\right.$ ' $-79^{\circ} 59^{\prime} \mathrm{W}$ y $2^{\circ} 02$ '$2{ }^{\circ} 18$ ' S) (Johansson et al., 2018) y cuenta con una población de 2,56 millones, aproximadamente (Alcaldía de Guayaquil, 2016) . El clima de Guayaquil se considera tropical húmedo, con una temperatura media anual de $26^{\circ} \mathrm{C}$ y una

\section{MAPA DE UBICACIÓN DE PUNTOS DE MUESTREO}

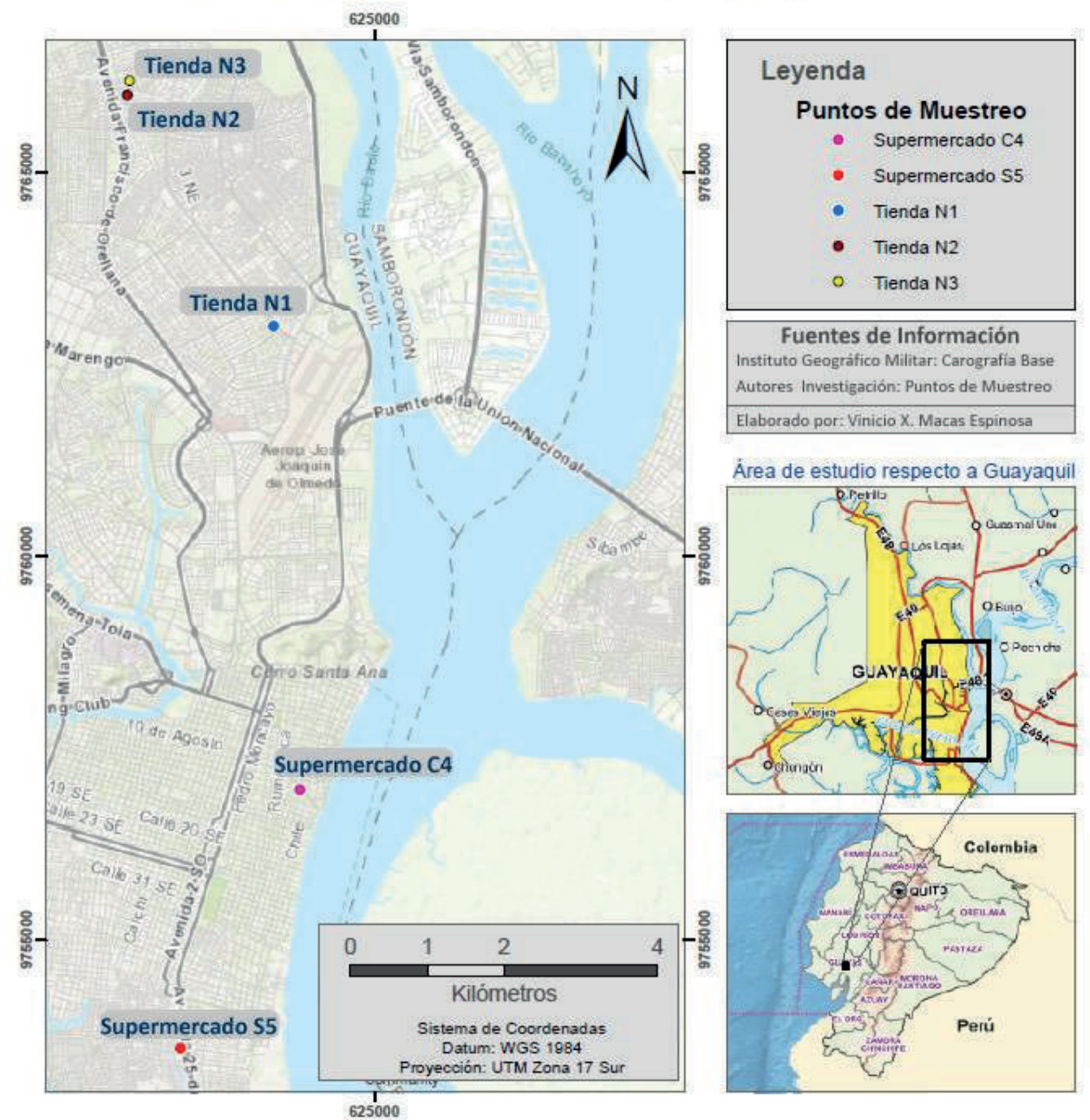

Figura 1. Mapa de los sitios de muestreo. 
precipitación media de $1030 \mathrm{~mm}$ (Johansson et al., 2018).

Dentro de la ciudad las muestras de chocolate en polvo se adquirieron en diferentes tiendas, mercados $y$ supermercados en el Norte-Centro-y Sur de la ciudad de Guayaquil en el mes de junio del año 2020 (Figura 1).

\section{Selección de las Muestras de Chocolate en Polvo}

La presente investigación es de tipo exploratoria. Para el análisis de $\mathrm{Cd}$ se adquirió tres marcas de chocolate en polvo dentro de las más consumidas en Ecuador según Rueda (2015). Se tomaron nueve muestras por marca $\mathrm{y}$ localidad, en supermercados y tiendas del norte, centro y sur de la ciudad mediante el método de muestreo probabilístico de tipo al azar simple (Tabla 1). Se analizó las tres zonas de la ciudad de Guayaquil para tener una representación espacial sobre la red de comercialización del chocolate en polvo y para verificar si existían diferencias en los contenidos de metales por lotes y por zonas. Con respecto al muestreo en tiendas $\mathrm{y}$ supermercados se basó en la accesibilidad del producto hacia los consumidores, así como también la realidad socioeconómica de los sectores, donde las personas de menores recursos económicos recurren a las tiendas cercanas a sus viviendas para abaratar los costos de movilización. Por el contrario, las personas con mayores recursos suelen comprar en supermercados.

Para la adquisición de los productos se verificó la fecha de caducidad vigente y números de lote diferentes. Se realizó la preparación de las muestras según la Norma Técnica Ecuatoriana NTE INEN 537:2013 en el cual nos indica los parámetros para el muestreo de productos derivados del cacao. Una vez listas las muestras, estas fueron transportadas para su análisis al Laboratorio Acreditado por el Sistema de Acreditación Ecuatoriano.

Tabla 1 .

Codificación de las muestras de chocolate en polvo, precio, origen e ingredientes

\begin{tabular}{|c|c|c|c|c|c|}
\hline Marcas & Sector & $\begin{array}{c}\text { Número } \\
\text { de } \\
\text { muestras }\end{array}$ & Precio (\$) & Origen & Ingredientes \\
\hline \multirow{3}{*}{ Marca 1} & Norte & $\mathrm{n}=9$ & $0.947 \pm 0.131$ & \multirow[t]{3}{*}{ Ecuador } & \multirow{3}{*}{$\begin{array}{c}\text { Polvo de cacao, azúcar, carbonato de calcio, } \\
\text { lecitina de soya, vainillina, hierro, vitaminas A, } \\
\text { B1, B2, B3, B6, C, y D3 }\end{array}$} \\
\hline & Centro & $\mathrm{n}=9$ & $0.800 \pm 0.050$ & & \\
\hline & Sur & $\mathrm{n}=9$ & $0.917 \pm 0.126$ & & \\
\hline \multirow{3}{*}{ Marca 2} & Norte & $\mathrm{n}=9$ & $1.093 \pm 0.100$ & \multirow[t]{3}{*}{ Ecuador } & \multirow{3}{*}{$\begin{array}{c}\text { Polvo de cacao, maltodextrina, caramelo en } \\
\text { polvo, etilvainillina, vitaminas A, B1, B2, B3, } \\
\text { B6, C, D, pirofosfato férrico, sulfato de zinc, } \\
\text { aroma a cacao, }\end{array}$} \\
\hline & Centro & $\mathrm{n}=9$ & $1.020 \pm 0.159$ & & \\
\hline & Sur & $\mathrm{n}=9$ & $0.953 \pm 0.064$ & & \\
\hline \multirow{3}{*}{ Marca 3} & Norte & $\mathrm{n}=9$ & $1.747 \pm 0.131$ & \multirow[t]{4}{*}{ Colombia } & \multirow{4}{*}{$\begin{array}{l}\text { Cacao, azúcar, sal, carbonato de calcio, lecitina } \\
\text { de soya, carboximetilcelulosa sódica, extracto de } \\
\text { malta de cebada, leche, etilvainillina, hierro, } \\
\text { vitaminas A, B1, B2, B3, D3 }\end{array}$} \\
\hline & Centro & $\mathrm{n}=9$ & $1.88 \pm 0.100$ & & \\
\hline & Sur & $\mathrm{n}=9$ & $1.747 \pm 0.131$ & & \\
\hline Total & & $\mathrm{n}=81$ & & & \\
\hline
\end{tabular}

Fuente: Elaboración propia. 


\section{Procesamiento de las muestras}

Para la determinación de metales pesados, se aplicó el método de espectrofotometría de absorción atómica con horno de grafito (Perkin-Elmer AAnalyst 400), según el método de referencia EPA 3051/7061 para la determinación de cadmio y plomo; mientras que para arsénico se empleó el método de referencia PI_MP5 2013 (Deaker \& Maher 1999). Se mantuvo las condiciones ambientales generales durante la realización del ensayo, entre 15 y $30^{\circ} \mathrm{C}$ y humedad relativa entre 20 y $80 \%$.

Para la preparación los estándares y soluciones se utilizó agua desionizada $(18 \mathrm{M} \Omega / \mathrm{cm})$ de un sistema Milli-Q (Millipore). Para la digestión ácida en microondas de las muestras se pesó 0,200 $\mathrm{g}$ del chocolate en polvo y se añadió 5,000 mL utilizó ácido nítrico libre de metales pesados (67\%, HNO3, Merck) y $1 \mathrm{~mL}$ de peróxido de hidrógeno (30\%, $\mathrm{H} 2 \mathrm{O} 2$, Merck) y $\mathrm{Pd}$ (NO3)2 como modificador de matriz de As. Las curvas de calibración se realizaron a partir de soluciones estándar de plomo y cadmio (Accustandar) y se utilizó como material de referencia CRM033 con un porcentaje de recuperación del 95\%.

\section{Análisis estadísticos}

Para los análisis estadísticos y las gráficas se utilizó el programa $\mathrm{R}$ versión 4.0.2. Para verificar la normalidad de los datos se aplicó el test de KolmogorovSmirnov y la prueba de Levene para identificar la igualdad de varianza entre los grupos. Para comparar la concentración de arsénico, cadmio y plomo entre las marcas y zonas se utilizó una ANOVA de una vía con test a posteriori de Tukey $(\mathrm{P} \leq 0,05)$.

\section{Resultados}

Se determinó la concentración de arsénico, plomo y cadmio en chocolate en polvo comercializado en la ciudad de Guayaquil. El plomo no fue detectable en ninguna de las muestras (Figura 2). El As se encontró en bajos niveles en la Marca $1(0,030 \pm 0,021 \mathrm{mg} / \mathrm{kg})$ y la Marca 3 $(0,060 \pm 0,034 \mathrm{mg} / \mathrm{kg})$. Lo que indica que el $\mathrm{As} \mathrm{y}$ el $\mathrm{Pb}$ no representan un peligro para la salud de los consumidores.

Por el contrario, se detectó $\mathrm{Cd}$ en todas las marcas de chocolate con diferencias significativas entre las marcas $(\mathrm{F}=245,80$ y $\mathrm{P}<0,001) . \quad$ La Marca 1 presentó un promedio de $0,236 \pm 0,082$ $\mathrm{mg} / \mathrm{kg}$, con un valor atípico de 0,062 $\mathrm{mg} / \mathrm{kg}$; la Marca 2 tuvo una media de $0,169 \pm 0,066 \mathrm{mg} / \mathrm{kg}$ con un valor mínimo de $0,074 \mathrm{mg} / \mathrm{kg}$ y máximo de $0,260 \mathrm{mg} / \mathrm{kg}$, representando la marca con menor concentración del metal pesado y la única que no representa un riesgo para la salud de los consumidores. Por el contrario, en la Marca 3 se apreció la mayor concentración con $1,440 \pm 0,212$ $\mathrm{mg} / \mathrm{kg}$ con valores mínimos de 1,200 $\mathrm{mg} / \mathrm{kg}$ y máximos de $1,900 \mathrm{mg} / \mathrm{kg}$. En comparación con el Reglamento Bromatológico Nacional de Uruguay, que estipula como límite máximo permisible de $\mathrm{Cd}$ de $0,20 \mathrm{mg} / \mathrm{kg}$, la Marca 1 y la Marca 3 sobrepasan el límite, mientras que la Marca 2 se encuentra dentro del valor establecido. Por otro lado, con 


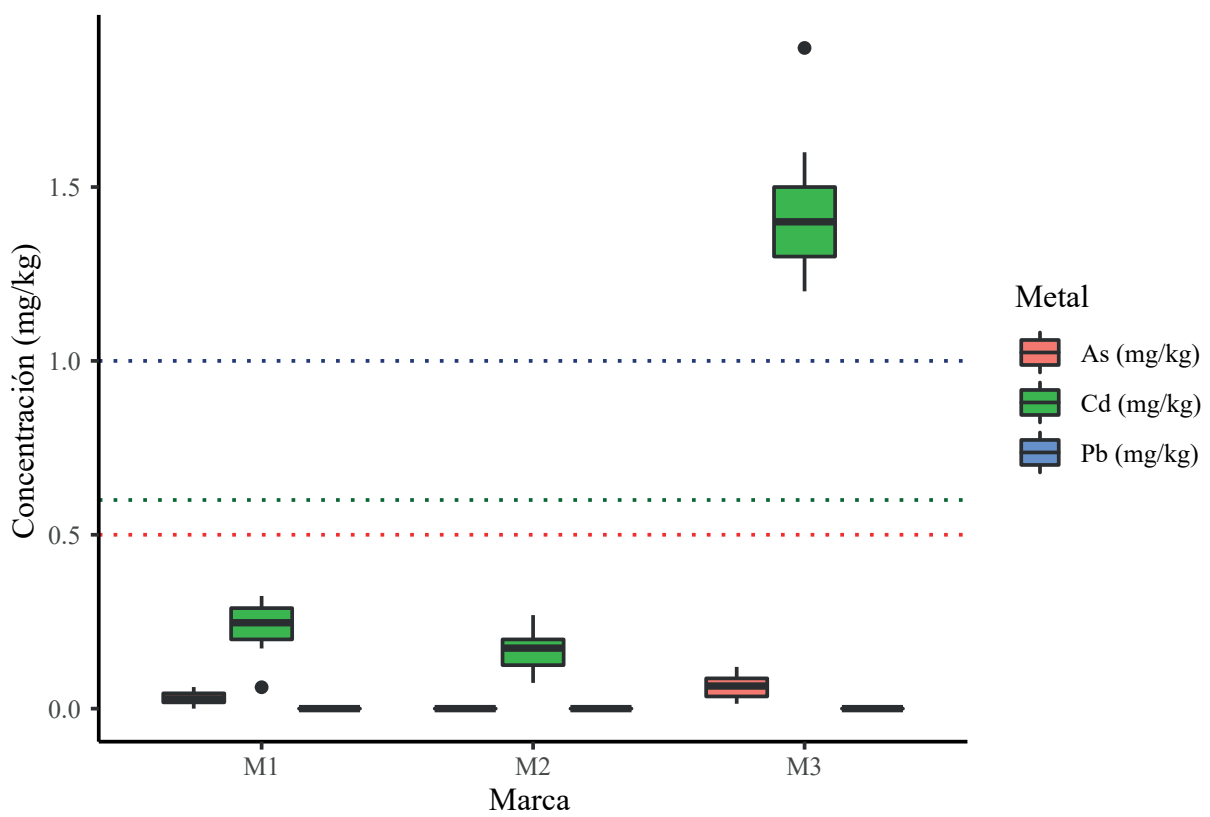

Figura 2. Concentraciones de $\mathrm{Cd}$ y $\mathrm{Pb}$ en las tres marcas analizadas. Los resultados se muestran como cajas y bigotes. Marca 1: M1, Marca 2: M2, Marca 3: M3; LMP (Pb): límite máximo permisible de plomo en chocolate según la norma INEN 621:2010 (línea azul); LMP (Cd): límite máximo permisible de cadmio según la Unión Europea (línea verde); LMP(As): límite máximo permisible de arsénico en chocolate según la norma INEN 621:2010 (línea roja). Letras iguales indican que no existen diferencias significativas según ANOVA de una vía y test a posteriori de Tukey $(\mathrm{P}>0,05)$.

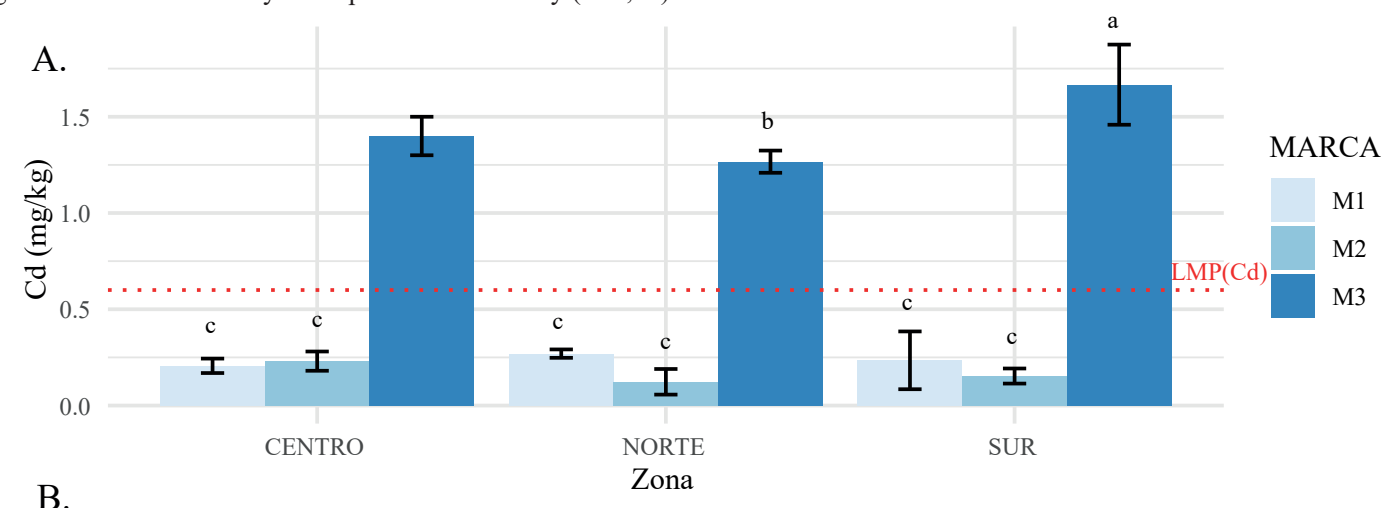

B

Zona

LMP(As)

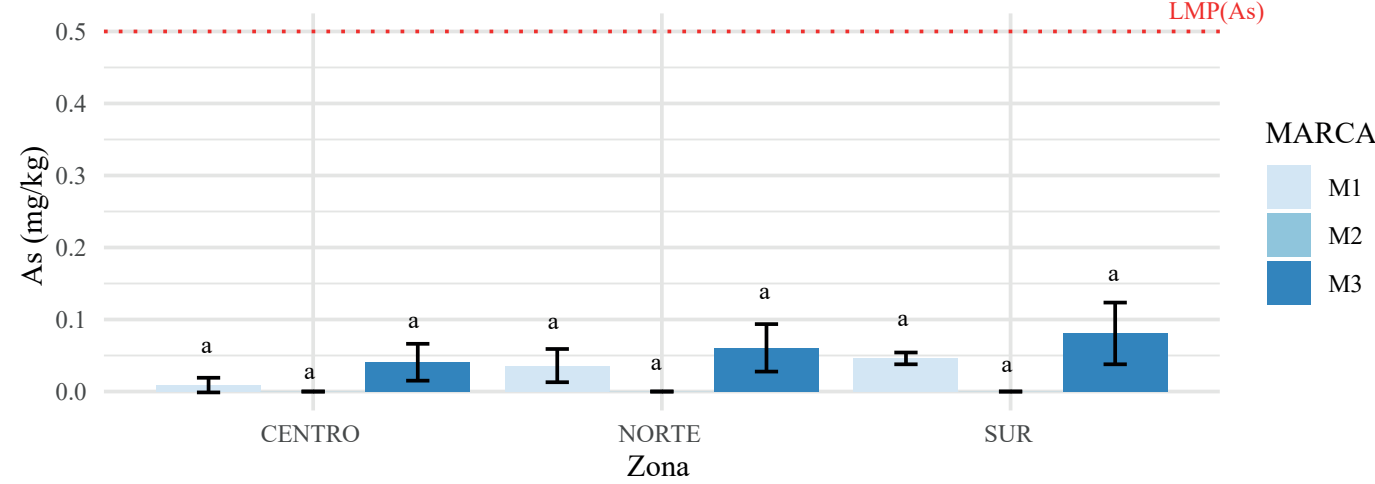

Figura 3. Concentración de A. Cadmio y B. Arsénico en las diferentes marcas de chocolate en polvo según la zona de la ciudad de Guayaquil donde fueron adquiridas. Marca 1: M1, Marca 2: M2, Marca 3: M3. LMP (Cd): límite máximo permisible de cadmio según la Unión Europea; LMP(As): límite máximo permisible de arsénico en chocolate según la norma INEN 621:2010. Letras iguales indican que no existen diferencias significativas según ANOVA de una vía y test a posteriori de Tukey $(\mathrm{P}>0,05)$. 
respecto a la Código de Normas Alimentarias (Food Standards Code) que sugiere un límite de $0,500 \mathrm{mg} / \mathrm{kg}$ de Cd y la norma de la Unión Europea $(0,600$ $\mathrm{mg} / \mathrm{kg}$ ), solo la Marca 3 supera todos los estándares, representado un riesgo para la salud de los consumidores.

Con respecto a la zona de la ciudad donde se expenden estos productos, en las Marcas 1 y 2 no se observaron diferencias significativas con respecto a la concentración de cadmio por zona $(\mathrm{P}>$ 0,05). En cambio, en la Marca 3 se observó una mayor concentración de cadmio en el chocolate expendido en los locales del Sur de la ciudad, donde se encuentran los barrios más pobres $(\mathrm{F}=$ 6,590; $\mathrm{P}=0,031)$. En esta marca los promedios variaron de $1,260 \pm 0,057$ $\mathrm{mg} / \mathrm{kg}$ en el norte de la ciudad a $1,670 \pm$
0,200 mg/kg en el sur, sin embargo, todos los valores se encontraron por encima de los límites admisibles, indicando que este chocolate no es apto para el consumo humano (Figura 3A). Por el contrario, el arsénico se halló en bajas concentraciones en todas las zonas de la ciudad, no se detectó en la Marca 2, ni se encontraron diferencias significativas entre las zonas $(\mathrm{P}>0,05)$. En la Marca 3 las concentraciones de As fueron de 0,060 \pm $0,033 \mathrm{mg} / \mathrm{kg}$ en la zona norte, $0,040 \pm 0,025 \mathrm{mg} / \mathrm{kg}$ en el centro y de $0,080 \pm 0,040 \mathrm{mg} / \mathrm{kg}$ en el sur de Guayaquil, inferiores al límite máximo permisible de 0,500 mg/kg (Figura 3B).

En la Figura 4 se aprecia una correlación lineal entre el Cd y el As con un valor de R2 de 0,450 y una correlación de Pearson de 0,670, indicando que, a

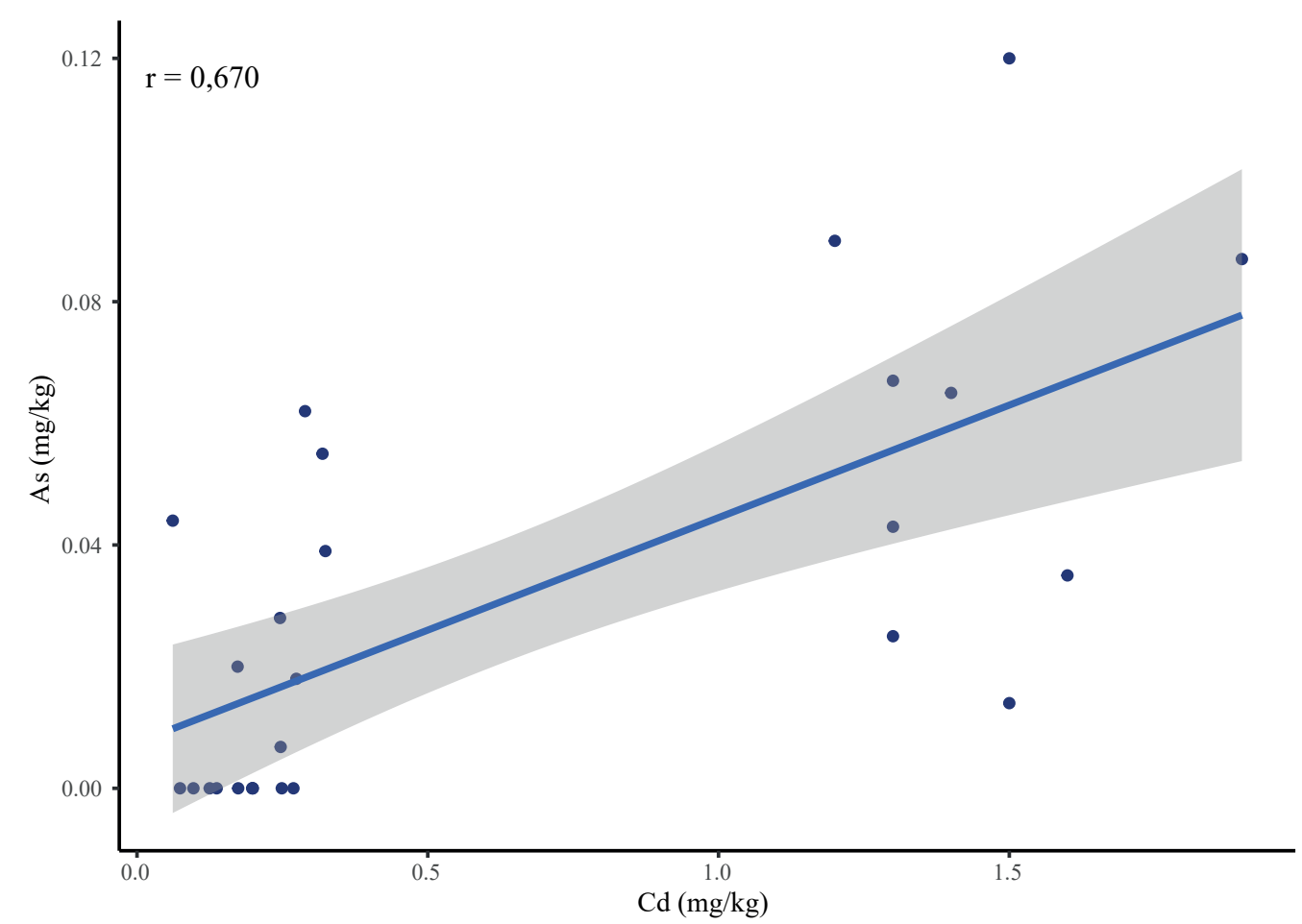

Figura 4. Correlación lineal entre la concentración de cadmio y arsénico en las muestras de chocolate en polvo comercializadas en la ciudad de Guayaquil. 


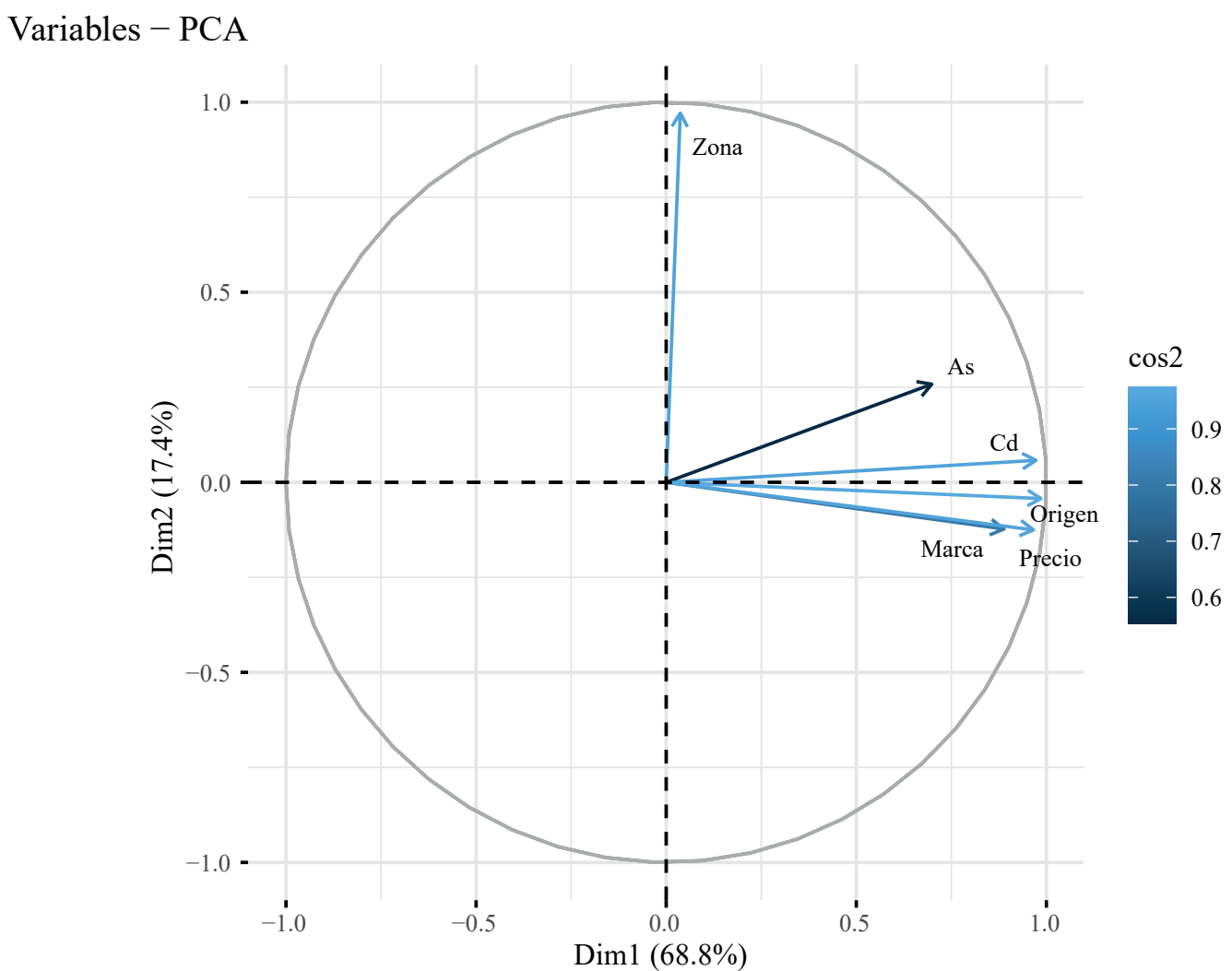

Figura 5. Análisis de componentes principales entre las variables concentración de cadmio (Cd), arsénico (As), el origen del chocolate en polvo, la marca, la zona de Guayaquil donde fue comercializado y el precio.

mayor nivel de cadmio, habrá mayor concentración de As en el chocolate en polvo comercializado en Guayaquil.

En la Figura 5 se aprecia un análisis de componentes principales donde se observó que cuatro de los componentes explicaron el $98,80 \%$ de la correlación entre las variables. El primer componente (Dim1) mostró la correlación entre la concentración de $\mathrm{Cd}$, As y el precio, origen y marca del chocolate en polvo, es decir, a mayor precio, mayor concentración de Cd y As. De igual manera, se observó correlación con el origen del chocolate, siendo mayor la concentración de $\mathrm{As}$ y $\mathrm{Cd}$ en el chocolate colombiano. Por el contrario, no hubo correlación entre la concentración de los metales por zonas de la ciudad de Guayaquil.

Comparativamente, en Ecuador los niveles de $\mathrm{Cd}$ en el chocolate en polvo son semejantes a los hallados en Estados Unidos, Malasia y Japón, menos elevados que Perú y Venezuela, y mayores a los encontrados en Italia, Polonia, Ghana, Brasil y Costa de Marfil (Tabla 2). Los niveles más bajos de plomo se hallaron en el presente estudio, donde no fue detectable y los valores más altos de plomo según la tabla 2 se encuentran en Brasil $(0,769 \pm 0,154$ $\mathrm{mg} / \mathrm{kg}$ ), seguido por Italia con $0,417 \pm$ $0,032 \mathrm{mg} / \mathrm{kg}$. En tanto As no se evidenciaron estudios previos en chocolate en polvo. 


\section{Discusión}

En el chocolate en polvo comercializado en Guayaquil no se evidenció contaminación por plomo ni arsénico. Estos resultados coinciden con los obtenidos por Barraza et al. (2018) y Barraza et al. (2021) quienes no encontraron plomo en cacao de Ecuador y contrario a los resultados de Mounicou et al. (2003) quienes hallaron 0,197 $\pm 0,000$ $\mathrm{mg} / \mathrm{kg}$ de plomo en cacao de origen ecuatoriano. Esta diferencia entre el año 2003 y 2020 podría deberse a una disminución en la contaminación por plomo como resultado de las medidas tomadas por el gobierno de eliminar el tetraetilo de plomo de la gasolina y prohibir el plomo como componente en las pinturas. También se debe considerar que la metodología empleada por Mounicou et al. (2003) fue espectrometría de masas con plasma acoplado inductivamente, a diferencia del presente estudio donde se utilizó espectrofotometría de absorción atómica con horno de grafito que tiene mayores límites de detección. Por el contrario, en otros países como Venezuela, Italia, Polonia, Ghana, Brasil y Costa de Marfil el chocolate en polvo contiene plomo (Mounicou et al., 2003; Alves Peixoto et al., 2018; Peixoto et al., 2016; Kruszewski et al., 2018; Lo Dico et al., 2018). Por otro lado, el As se encontró en bajas concentraciones, resultados que coinciden con los hallados en chocolate en polvo de Malasia donde encontraron $0,05 \pm 0,07 \mathrm{mg} / \mathrm{kg}$ (Mohamed et al., 2020 ) y de Italia $0,026 \pm 0,005 \mathrm{mg} / \mathrm{kg}$ (Lo Dico et al., 2018).

Con respecto al $\mathrm{Cd}$, en el chocolate en polvo se encontró este metal en todas marcas. La fuente principal de la contaminación del chocolate en polvo es el cacao (Mounicou et al., 2003; Yanus et al., 2014; Laila et al., 2019). En este sentido, se ha descrito altas concentraciones de $\mathrm{Cd}$ en semillas de cacao en numerosos

Tabla 2.

Comparación de la concentración de $\mathrm{Cd}$ y $\mathrm{Pb}$ promedio en chocolate en polvo de Ecuador con respecto a otros países

\begin{tabular}{|c|c|c|c|c|}
\hline País & $\mathrm{Cd}(\mathrm{mg} / \mathrm{kg})$ & $\mathrm{Pb}(\mathrm{mg} / \mathrm{kg})$ & Metodología & Autores \\
\hline Perú & $2,260 \pm 0.560$ & - & EAA -HG & Laila et al., 2019 \\
\hline Venezuela & $1,833 \pm 0,020$ & $0,173 \pm 0,012$ & ICP -MS & Mounicou et al., 2003 \\
\hline Ecuador & $0,738 \pm 0,053$ & $0,197 \pm 0,000$ & ICP -MS & Mounicou et al., 2003 \\
\hline Estados Unidos & $0,700 \pm 0,830$ & $0,110 \pm 0,100$ & ICP -MS & Abt et al., 2018 \\
\hline Ecuador & $0,610 \pm 0,600$ & $0,000 \pm 0,000$ & EAA -HG & Presente estudio \\
\hline Malasia & $0,602 \pm 0,073$ & $0,227 \pm 0,069$ & ICP -MS & Mounicou et al., 2003 \\
\hline Ecuador El Oro & $0,533 \pm 0,016$ & $0,011 \pm 0,003$ & ICP -MS & Mounicou et al., 2003 \\
\hline Malasia & $0,330 \pm 0,260$ & $0,270 \pm 0,220$ & ICP -MS & Mohamed et al., 2020 \\
\hline Japón & $0,320 \pm 0.380$ & - & ICP -MS & Yohei et al., 2018 \\
\hline Italia & $0,159 \pm 0.020$ & $0,417 \pm 0,032$ & ICP -MS & Lo Dico et al., 2018 \\
\hline Polonia & $0,153 \pm 0,015$ & $0,575 \pm 0,014$ & EAA -HG & Kruszewski et al., 2018 \\
\hline Ghana & $0,133 \pm 0,020$ & $0,052 \pm 0,010$ & ICP -MS & Mounicou et al., 2003 \\
\hline Brasil & $0,124 \pm 0,003$ & $0,150 \pm 0,009$ & EAA -HG & $\begin{array}{l}\text { Alves Peixoto et al., } \\
2018 \text {; Peixoto et al., } 2016\end{array}$ \\
\hline Brasil & $0,081 \pm 0,001$ & $0,769 \pm 0,154$ & ICP -MS & Mounicou et al., 2003 \\
\hline Costa de Marfil & $0,054 \pm 0,014$ & $0,191 \pm 0,039$ & ICP -MS & Mounicou et al., 2003 \\
\hline
\end{tabular}

Nota: Los resultados se muestran como media \pm desviación estándar. ICP-MS: Espectrometría de Masas con Plasma Acoplado Inductivamente; EAA-HG : Espectrofotometría de absorción atómica con horno de grafito.

Fuente: Ministerio del Trabajo (2017). Elaboración propia. 
trabajos realizados en Ecuador donde los niveles van desde no detectables hasta $10,400 \mathrm{mg} / \mathrm{kg}$ de $\mathrm{Cd}$ (Argüello et al., 2019; Barraza et al., 2017; Chavez et al., 2015; Mite et al., 2010). Y, según Barraza et al. (2017) la acumulación de este metal en las plantas, se debe a la movilidad del cadmio en suelos y su absorción depende de varios factores tales como: la textura del suelo, la capacidad de intercambio catiónico, el pH, el contenido de materia orgánica total en los suelos, la especiación química, las variedades $\mathrm{y}$ especies de plantas, y a las prácticas agrícolas. Lo que nos indica que, los suelos en los que se cultiva el cacao presentan las condiciones óptimas para que el $\mathrm{Cd}$ se transfiera al fruto y se encuentre en grandes concentraciones que afectan la calidad del chocolate y de los productos elaborados a base de cacao. En este sentido, las diferencias halladas entre las marcas de chocolate podrían deberse a que cada empresa tiene sus distribuidores de cacao, mismos que provienen de diferentes zonas de Ecuador y por lo descrito, dependiendo del área y el tipo de suelo y variedad de cacao, la materia prima puede contener mayores concentraciones del metal pesado (Argüello et al., 2019; Barraza et al., 2017). La diferencia entre las marcas también podría deberse a los procesos de fabricación que influyen en la concentración de metales (Yanus et al., 2014).

En cuanto a las diferencias en la concentración de cadmio en Marca 3 en las diferentes zonas de la ciudad podría deberse a que el lote analizado fue diferente en cada zona, lo que indicaría que los lotes de cacao contienen diferentes concentraciones de cadmio. En este sentido, la empresas compran el cacao a diferentes proveedores para satisfacer sus requerimientos y es posible que algunos proveedores presenten mayores concentraciones de cadmio en sus suelos, lo que se refleja en un cacao con mayor contaminación por cadmio. También podría deberse a una discrepancia en la calidad del producto que se expende entre las zonas más humildes de la ciudad (zona sur) y aquellas donde existe un control de calidad más estricto (zona norte).

Otra posible fuente de contaminación por metales pesados en el chocolate en polvo son los otros ingredientes añadidos tales como el azúcar (Alcívar y Pernía, 2019), la leche (Pernía et al., 2015; Zhou et al., 2019), y el extracto de malta (Chen et al., 2007; Čejka et al., 2011; Gramss et al., 2020). En este sentido coincide que la Marca 3 que contenía mayores concentraciones de Cd contenía leche y extracto de malta. De allí se recomienda para futuras investigaciones analizar las concentraciones de $\mathrm{Cd}$ en los aditivos del chocolate.

Los valores hallados en el chocolate en polvo del presente estudio fueron muy elevados en comparación con los datos obtenidos en el Salvador por Beltrán et al. (2017) donde encontraron niveles de cadmio en las muestras de chocolate en un rango de 0,003 a $0,018 \mathrm{mg} / \mathrm{kg}$. También fueron mayores a los valores de cadmio hallados en otros países como Italia, Polonia, Ghana, Brasil y Costa de Marfil. Pero, los resultados del chocolate en polvo fueron menores a los hallados en

Perú con 2,260 $\pm 0,560 \mathrm{mg} / \mathrm{kg}$ (Laila 
et al., 2019) y Venezuela $1,833 \pm 0,020$ $\mathrm{mg} / \mathrm{kg}$ (Mounicou et al., 2003). Estas diferencias entre países podrían deberse a las discrepancias entre las concentraciones de metales pesados en los suelos de los diferentes países, al uso de distintas variedades de cacao ya que, según Arévalo-Hernández et al. (2020) este es una factor determinante debido a que existen variedades de cacao que absorben menores concentraciones de $\mathrm{Cd}$ tales como AYP-22, PAS-105, UGU-126, ICT-1026, ICT-1087, ICT-1189, ICT-1292, PH-17, CCN-51, ICS-39 and TSH-565, mientras que, otras variedades hiperacumulan altas concentraciones. Otra posible razón es el uso de fertilizantes fosfatados con altos contenidos de cadmio en países donde estos no son regulados como en el caso de Ecuador, donde no existen límites máximos permisibles de metales pesados en los fertilizantes y a las prácticas agronómicas como lo indica Arguello et al. (2019).

Sin embargo, llama la atención que en todos los países productores de cacao se evidencia la presencia del cadmio en el chocolate en polvo, lo que representa una fuente de contaminación en especial para los niños que les encantan las bebidas achocolatadas y que representan una fuente importante de calcio en su dieta diaria (Thompson et al., 2007; Fayet-Moore, 2016).

Es importante mencionar que el Cd se acumula en la cadena alimentaria a través de los productos del cacao, las consecuencias en los niños, que son los principales consumidores de chocolates, incluyen morbilidad y mortalidad (Maddela et al., 2020). Los efectos que el
Cd podría generar en los consumidores de chocolate en polvo son daño renal, osteoporosis, fracturas óseas dolorosas, diabetes, anemia y cáncer (Laila et al., 2019; ATSDR, 2020) y en los niños además podría producir alteraciones en el comportamiento y aprendizaje (ATSDR, 2020). Es por ello, que se deben tomar medidas por parte de las autoridades para proteger la salud de los consumidores de chocolate en polvo en Ecuador y especialmente para salvaguardar la salud de los niños.

Por otro lado, en la presente investigación se observó correlación entre la concentración de $\mathrm{Cd}$ y As en el chocolate en polvo, indicando que ambos contaminantes podrían derivar de una misma fuente, por ejemplo, del uso de fertilizantes fosfatados en los suelos de cultivo del cacao. También se apreció una correlación positiva entre la concentración de $\mathrm{Cd}$ y el precio del producto, indicando que, a mayor precio, mayor concentración de este metal; $y$, a su vez, el precio se correlacionó con la marca y el origen, siendo la marca más costosa la de origen colombiano, probablemente por los aranceles de importación del producto. Es preocupante que la marca de chocolate en polvo que superó los límites permitidos es de origen colombiano, lo que demuestra una escasa vigilancia de los niveles de metales pesados en productos importados. Así mismo, también es preocupante que la norma INEN 621: 2010 regula los metales pesados $\mathrm{As}, \mathrm{Cu}, \mathrm{Pb}$ e chocolate y no el $\mathrm{Cd}$.

Este estudio representa una línea base de alerta sanitaria por lo que se 
recomienda a las autoridades de salud llevar a cabo un estudio en todo Ecuador donde se analicen las concentraciones de cadmio en todas las marcas de chocolate en polvo a nivel nacional con un mayor número de réplicas y se incluya dentro de la norma INEN 621:2010 un límite máximo permisible para $\mathrm{Cd}$ en chocolate.

Dentro de las medidas que podrían tomarse para asegurar la inocuidad alimentaria en el consumo de derivados del cacao son: i) desarrollo de clones de cacao con una capacidad innata de absorber niveles bajos de $\mathrm{Cd}$ de los suelos (Maddela et al., 2020); ii) evitar el cultivo de cacao en suelos que superen los 0,500 $\mathrm{mg} / \mathrm{kg}$ de $\mathrm{Cd}$; iii) para reducir el contenido de $\mathrm{Cd}$ en el suelo de los cultivos se propone el uso de fitorremediación con especies de plantas que acumulan $\mathrm{Cd}$ en sistemas agroforestales (Laila et al., 2019); iv) evitar el uso de fertilizantes fosfatados, v) añadir materia orgánica y microelementos al suelo para evitar la absorción del metal pesado; vi) regular el $\mathrm{pH} ; \mathrm{y}$, finalmente, vii) desarrollar una norma nacional de cumplimiento obligatorio donde se limite el contenido de $\mathrm{Cd}$ en chocolate en polvo.

\section{Agradecimientos}

Los autores agradecemos al Proyecto Derek FCI-029 2019 de la Universidad de Guayaquil.

\section{Referencias}

Abt, E., Fong Sam, J., Gray, P., \& Robin, L. P. (2018). Cadmium and lead in cocoa powder and chocolate products in the US Market.
Food Additives and Contaminants: Part B Surveillance, 11(2), 92-102. https://doi. org/10.1080/19393210.2017.1420700

Alcaldía de Guayaquil. (2016). Guayaquil-Puerto Principal de Ecuador. https://guayaquil. gob.ec/noticias-actuales/246

Alcívar, M., \& Pernía, B. (2019). Determinación de cadmio y plomo en productos derivados de la caña: azúcar blanca, morena y panela, comercializados en Ecuador. $V$ Congreso Iberoamericano Sobre Ambiente y Sustentabilidad, 14-15

Alves Peixoto, R. R., Oliveira, A., \& Cadore, S. (2018). Risk assessment of cadmium and chromium from chocolate powder. Food Additives and Contaminants: Part $B$ Surveillance, 11(4), 256-263. https://doi. org/10.1080/19393210.2018.1499676

Anecacao. (2015). Asociación Nacional de Exportadores de Cacao-Ecuador. http://www.anecacao.com/index.php/es/e stadisticas/estadisticas-actuales.html.

Ahn, J., Park, M. Y., Kang, M. Y., Shin, I. S., An, S., \& Kim, H. R. (2020). Occupational lead exposure and brain tumors: Systematic review and meta-analysis. International Journal of Environmental Research and Public Health, 17(11), 1-14. https://doi.org/10.3390/ijerph17113975

Anyimah-Ackah, E., Ofosu, I. W., Lutterodt, H. E., \& Darko, G. (2019). Exposures and risks of arsenic, cadmium, lead, and mercury in cocoa beans and cocoa-based foods: A systematic review. Food Quality and Safety, 3(1), 1-8. https://doi.org/10.1093/ fqsafe/fyy 025

Arévalo-Hernández, C. O., Arévalo-Gardini, E., Farfán, A., He, Z., \& Baligar, V. C. (2020). Growth and nutritional responses of wild and domesticated cacao genotypes to soil cd stress. Science of the Total Environment, 763, 144021. https:// doi.org/10.1016/j.scitotenv.2020.144021 
Argüello, D., Chavez, E., Lauryssen, F., Vanderschueren, R., Smolders, E., \& Montalvo, D. (2019). Soil properties and agronomic factors affecting cadmium concentrations in cacao beans: A nationwide survey in Ecuador. Science of the Total Environment, 649, 120-127. https://doi. org/10.1016/j.scitotenv.2018.08.292

ATSDR. (2020). Resúmenes de Salud Pública-Cadmio https://www.atsdr.cdc.gov/es/phs/es_phs 5.html\#: :text=En $\% 20$ general $\% 20$ no $\% 2$ 0se $\% 20$ han,m\%C3\%A1s $\% 20$ cadmio $\% 2$ 0que $\% 201$ os $\% 20$ adultos

Barraza, F, Schreck, E., Leveque, T., Uzu, G., López, F., Ruales, J., Prunier, J., Marquet, A., \& Maurice, L. (2017). Cadmium bioaccumulation and gastric bioaccessibility in cacao : A field study in areas impacted by oil activities in Ecuador. Environmental Pollution Journal, 229, 950-963. https:// doi.org/10.1016/j.envpol.2017.07.080

Barraza, F., Maurice, L., Uzu, G., Becerra, S., López, F., Ochoa-Herrera, V., Ruales, J., \& Schreck, E. (2018). Distribution, contents and health risk assessment of metal(loid)s in small-scale farms in the Ecuadorian Amazon: An insight into impacts of oil activities. Science of the Total Environment, 622-623, 106-120. https://doi.org/10.1016/j.scitotenv.2017.11.246

Barraza, F., Schreck, E., Uzu, G., Lévêque, T., Zouiten, C., Boidot, M., \& Maurice, L. (2021). Beyond cadmium accumulation: Distribution of other trace elements in soils and cacao beans in Ecuador. Environmental Research, 192, 110241. https://doi.org/10.1016/j.envres.2020.110241

Beltrán, E., Hernández, K., \& Rodríguez, A. (2017). Determinación de cadmioen chocolate de mesa comercializado en la zona metropolitana de San Salvador. San Salvador: Universidad de El Salvador. Obtenido de http://ri.ues.edu.sv/id/eprint/ 13124/1/Determinaci\%C3\%B3n\%20de\% 20cadmio $\% 20$ en $\% 20$ chocolate $\%$.pdf

Bertoldi, D., Barbero, F., Caligiani, A. \& Larcher, R. (2016). Multielemental fingerprinting and geographic traceability of Theobroma cacao beas and cocoas products. Food Control, 65, 46-53. https://doi.org/10.10 16/j.foodcont.2016.01.013

Casteblanco, J. A. (2018). Heavy metals remediation with potential application in cocoa cultivation. Granja, 27(1), 21-35. https://doi.org/10.17163/lgr.n27.2018.02

Čejka, P., Horák, T., Dvořák, J., Čulík, J., \& Jurková, M. (2011). Monitoring of the distribution of some heavy metals during brewing process. Ecol Chem Engineering, 18, 67-74.

Chavez, E., He, Z. L., Stoffella, P. J., Mylavarapu, R. S., Li, Y. C., Moyano, B., \& Baligar, V. C. (2015). Concentration of cadmium in cacao beans and its relationship with soil cadmium in southern Ecuador. Science of the Total Environment, 533, 205-214. https://doi.org/10.1016/j.scitotenv.2015.0 6.106

Chavez, E., He, Z. L., Stoffella, P. J., Mylavarapu, R. S., Li, Y. C., \& Baligar, V. C. (2016). Chemical speciation of cadmium: An approach to evaluate plant-available cadmium in Ecuadorian soils under cacao production. Chemosphere, 150. https:// doi.org/10.1016/j.chemosphere.2016.02.013

Chen, F., Dong, J., Wang, F., Wu, F., Zhang, G., Li, G., Chen, Z., Chen, J., \& Wei, K. (2007). Identification of barley genotypes with low grain $\mathrm{Cd}$ accumulation and its interaction with four microelements. Chemosphere, 67, 2082-2088. https:// doi.org/10.1016/j.chemosphere.2006.10.014

Chen, X., Zhu, G., Wang, Z., Zhou, H., He, P., Liu, Y., \& Jin, T. (2019). Ecotoxicology and Environmental Safety The association between lead and cadmium co-exposure and renal dysfunction. Ecotoxicology and Environmental Safety, 173, 429-435. 
https://oi.org/10.1016/j.ecoenv.2019.01.121

De Souza, P. A., Moreira, L. F., Sarmento, D. H. A., \& da Costa, F. B. (2018). CacaoTheobroma cacao. In Exotic Fruits (pp. 69-76). Elsevier. https://doi.org/10.1016/ b978-0-12-803138-4.00010-1

Deaker, M., and Maher, W. (1999). Determination of arsenic in arsenic compounds and marine biological tissues using low volume microwave digestion and electrothermal atomic absorption spectrometry. J. Anal. At. Spectrom. 14, 1193-1207. https://doi: 10.1039/a903790j

Devóz, P. P., Gomes, W. R., Araújo, M. L. De, Ribeiro, L., Pedron, T., Maria, L., Antunes, G., Lemos, B., Jr, F. B., Rafael, G., Barcelos, M., Ribeiro, D. L., Pedron, T., Maria, L., Antunes, G., Batista, B. L., Jr, F. B., Rafael, G., Barcelos, M., \& Pb, L. (2017). Lead $(\mathrm{Pb})$ exposure induces disturbances in epigenetic status in workers exposed to this metal. Journal of Toxicology and Environmental Health, Part A, 00(00), 1-8. https://doi.org/ 10.1080/15287394.2017.1357364

FAO, Organización de las Naciones Unidas para la Alimentación y la Agricultura. (2018). FAOSTAT. http://www.fao.org/faostat/en/\#home

Fayet-Moore, F. (2016). Effect of flavored milk vs plain milk on total milk intake and nutrient provision in children. Nutrition Reviews, 74(1), 1-17. https://doi.org/10. 1093/nutrit/nuv031

Flora, S., \& Agrawal, S. (2017). Arsenic, Cadmium, and Lead. In R. Gupta (Ed.), Reproductive and Developmental Toxicology (2nd ed., pp. 537-566). Elsevier Inc. https://doi.org/10.1016/ B978-0-12-804239-7.00031-7

Furcal-Beriguete, P., \& Torres-Morales, J. L. (2020). Determinación de concentraciones de cadmio en plantaciones de Theobroma cacao L. en Costa Rica. Revista
Tecnología En Marcha, 33, 122-137. https://doi.org/10.18845/tm.v33i1.5027

García, P., Esmeralda, P., Cruz, A., \& Isabel, M. (2012). Los efectos del cadmio en la salud. Revista de Especialidades Médico-Quirúrgicas, 17(3), 199-205.

Gramss, G. (2020). Control of Heavy Metals from Barley and Wheat Grains during Malting and Brewing. Advances in Nutrition and Food Science, 2020(05). https://doi.org/ 10.37722/ANAFS.20205

Hamid, Y., Tang, L., Sohail, M. I., Cao, X., Hussain, B., Aziz, M. Z., Usman, M., He, Z. li, \& Yang, X. (2019). An explanation of soil amendments to reduce cadmium phytoavailability and transfer to food chain. Science of the Total Environment, 660, 80-96. https://doi.org/10.1016/j. scitotenv.2018.12.419

Huamani, H., Huauya, M.A., Mansilla, L., Florida, N. \& Neia, G. (2012). Presence of heavy metals in organic cacao (Theobroma cacao L.) crops. Acta Agron, 61 (4), 339-344.

Johansson, E., Yahia, M. W., Arroyo, I., \& Bengs, C. (2018). Outdoor thermal comfort in public space in warm-humid Guayaquil, Ecuador. International Journal of Biometeorology, 62(3), 387-399. https:// doi.org/10.1007/s00484-017-1329-x

Khan, K. M., Chakraborty, R., Bundschuh, J., Bhattacharya, P., \& Parvez, F. (2020). Health effects of arsenic exposure in Latin America: An overview of the past eight years of research. Science of the Total Environment, 710, 136071. https:// doi.org/10.1016/j.scitotenv.2019.136071

Kruszewski, B., Obiedziński, M. W., \& Kowalska, J. (2018). Nickel, cadmium and lead levels in raw cocoa and processed chocolate mass materials from three different manufacturers. Journal of Food Composition and Analysis, 66, 127-135. https://doi.org/10.1016/j.jfca.2017.12.012 
Laila, K., Zug, M., Alfredo, H., Yupanqui, H., Meyberg, F., Cierjacks, J. S., \& Cierjacks, A. (2019). Cadmium Accumulation in Peruvian Cacao (Theobroma cacao L.) and Opportunities for Mitigation. Water Air Soil Pollut, 230, 72. https://doi.org/ 10.1007/s11270-019-4109-x

Lo Dico, M., Galvano, F., Dugo, G., Carlo, D., Macaluso, A., Vella, A., Giangrosso, G., Cammileri, G., \& Ferrantelli, V. (2018). Toxic metal levels in cocoa powder and chocolate by ICP-MS method after microwave-assisted digestion. Food Chemistry, 245, 1163-1168. https://doi. org/10.1016/j.foodchem.2017.11.052

Maddela, N. R., Kakarla, D., García, L. C., Chakraborty, S., Venkateswarlu, K., \& Megharaj, M. (2020). El cadmio cargado de cacao amenaza la salud humana y la economía del cacao: una visión crítica. Science of the Total Environment, 137645. https:// doi.org/10.1016/j.scitotenv.2020.137645

Maiti, S., Chattopadhyay, S., Deb, B., Samanta, T., Maji, G., Pan, B., Ghosh, A., \& Ghosh, D. (2012). Antioxidant and metabolic impairment result in DNA damage in arsenic-exposed individuals with severe dermatological manifestations in Eastern India. Environmental Toxicology, 27(6), 342-350. https://doi.org/10.1002/tox.20647

Mandal, B. K., \& Suzuki, K. T. (2002). Arsenic round the world: A review. Talanta, 58(1), 201-235. https://doi.org/10.1016/S00399140(02)00268-0

Mite, F., Carrillo, M., \& Durango, W. (2010). Avances del monitoreo de Presencia de cadmio en almendras de cacao, suelo y agua en Ecuador. XII Congreso Ecuatoriano de La Ciencia Del Suelo XII Congreso Ecuatoriano de La Ciencia Del Suelo, 17-19.

Mohamed, R., Zainudin, B. H., \& Yaakob, A. S. (2020). Method validation and determination of heavy metals in cocoa beans and cocoa products by microwave assisted digestion technique with inductively coupled plasma mass spectrometry. Food Chemistry, 303, 125392. https://doi.org/ 10.1016/j.foodch em.2019.125392

Mounicou, S., Szpunar, J., Andrey, D., Blake, C., \& Lobinski, R. (2003). Concentrations and bioavailability of cadmium and lead in cocoa powder and related products. Food Additives and Contaminants, 20(4), 343-352. https://doi.org/10.1080/026520 3031000077888

Peixoto, R. R. A., Devesa, V., Vélez, D., Cervera, M. L., \& Cadore, S. (2016). Study of the factors influencing the bioaccessibility of 10 elements from chocolate drink powder. Journal of Food Composition and Analysis, 48, 41-47. https:// doi.org/10. 1016/j.jfca.2016.02.002

Pernía, B., Mero, M., Bravo, K., Ramírez, N., Lopez, D., Muñoz, J., \& Egas, F. (2015). Detección de cadmio y plomo en leche de vaca comercializada en la ciudad de Guayaquil , Ecuador. Revista Cientifica de Ciencias Naturales y Ambientales, 8 (2), 81-86 https://www.academia.edu/43 077535/Detección_de_cadmio_y_plomo en_leche_de_vaca_comercializada_en_1 a_ciudad_de_Guayaquil_Ecuador

Rueda, J. (2015). Proyecto de factibilidad para la elaboración y comercialización de chocolate de cacao en polvo fluido, en el cantón Huaquillas provincia Del Oro. Tesis de Ingeniera Comercial. Universidad Nacional de Loja, Ecuador.

Thompson, J. L., Gerard, P. D., \& Drake, M. A. (2007). Chocolate milk and the Hispanic consumer. Journal of Food Science, 72(9), S666-S675. https://doi.org/10.1111 /j.1750-3841.2007.00559.x

Yohei, K., Takahiro, W., Kyoko, H., \& Hiroshi, A. (2018). Surveillance of Cadmium Concentration in Chocolate and Cocoa Powder Products Distributed in Japan. Food Hyg. Saf. Sci. Vol., 59(6), 269-274. https://doi.org/10. 3358/shokueishi.59.269 